\title{
Descripción de tres especies nuevas del género Drosophila (Diptera, Drosophilidae) en el Ecuador
}

\author{
María Luna Figuero \& Violeta Rafael
}

Laboratorio de Genética Evolutiva, Escuela de Ciencias Biológicas, Pontificia Universidad Católica del Ecuador, 17-01-2184, Quito, Ecuador (lunaf3@hotmail.com; vrafael@puce.edu.ec).

\begin{abstract}
Description of three new species of Drosophila (Diptera, Drosophilidae) in Ecuador. Three new species of Drosophila were found among individuals collected in different areas of Ecuador. One of them belongs to the Drosophila willistoni group, other to D. asiri group and a third species is not assigned to any group. All samples were taken using plastic bottles containing banana and yeast. Species mentioned are: D. (Sophophora) neocapnoptera sp. nov., similar to Drosophila capnoptera Patterson \& Mainland, 1944, but showing differences in wing issues. Drosophila (Drosophila) neoasiri sp. nov., similar to D. asiri Vela \& Rafael, 2005, with a significant difference at the aedeagus and Drosophila (D.) papallacta sp. nov. not related to any group of species of Drosophila.
\end{abstract}

KEYWORDS. Drosophila asiri group, Drosophila willistoni group, Neotropical, Taxonomy.

RESUMEN. Se encontraron tres especies nuevas de Drosophila entre los individuos colectados en diferentes localidades del Ecuador. Una de las especies nuevas pertenecen al grupo Drosophila willistoni y otra al grupo Drosophila asiri, la tercera especie se encuentra sin agrupar. En todos los muestreos realizados se usaron trampas fabricadas con botellas de plástico agujereadas con cebo de banano y levadura. Las tres especies son: D. (Sophophora) neocapnoptera sp. nov., esta especie es similar a D. capnoptera Patterson \& Mainland, 1944, sin embargo presentan algunas diferencias en el ala que permiten distinguirlas. Drosophila (Drosophila) neoasiri sp. nov., una especie similar a D. asiri Vela \& Rafael, 2005, la diferencia más relevante entre las dos especies se observa a nivel del edeago y Drosophila (Drosophila) papallacta sp. nov. que por el momento no se encuentra relacionada a ningún grupo de especies del género Drosophila.

PALABRAS-CLAVE. Grupo Drosophila asiri, Grupo Drosophila willistoni, Neotropical, Taxonomía.

El género Drosophila, con 1.146 especies distribuidas alrededor del mundo (BraKe \& BäCHLI, 2008), está dividido en nueve subgéneros, entre ellos Sophophora con 300 especies aproximadamente (O’Grady \& Kidwell, 2002) y Drosophila con más de 700 especies (BRAKE \& BäCHLI, 2008).

En el Ecuador el subgénero Sophophora ha sido poco estudiado, sin embrago se conoce la existencia de 21 especies del subgénero en el país divididas en cuatro grupos de especies: D. melanogaster (cinco especies), D. obscura (una especie), D. saltans (siete especies) y $D$. willistoni (ocho especies) distribuido en varias provincias del Ecuador, desde el nivel del mar hasta los $1.800 \mathrm{~m}$ (ACurio \& RAFAel, 2009). Estos subgrupos fueron propuestos por STURTEVANT (1939, 1942), sin embargo, posteriormente se añadieron tres subgrupos que no se han encontrado en el país ya que son principalmente de origen Australiano y Afrotropical; estos son $D$. dispar, D. fima y D. dentissima (O'GradY \& KIDWELL, 2002).

Por otro lado, el subgénero Drosophila ha sido ampliamente estudiado en el Ecuador. Hasta el momento se han reportado 125 especies aproximadamente del subgénero en el país, divididas en 17 grupos de especies; los más representativos son el grupo $D$. repleta (28 especies) y el grupo D. tripunctata (24 especies) (Acurio \& Rafael, 2009; Figuero \& Rafael, 2011; Figuero et al., 2012a,b; CÉSPEdes \& RAFAel, 2012). FigUero et al. (2012) proponen un nuevo grupo de especies del subgénero Drosophila, denominado grupo $D$. asiri exclusivo de los Andes ecuatorianos, que por el momento agrupa a tres especies.
Entre los individuos de Drosophila colectados en los años 2006, 2010 y 2012 en diferentes localidades del país, se encontraron tres especies nuevas para la ciencia. Una de ellas perteneciente al subgénero Sophophora del grupo $D$. willistoni; esta especie nueva es la primera del subgénero y del grupo descrita en el Ecuador. Otra de las especies nuevas pertenece al subgénero Drosophila del grupo $D$. asiri, y por último una especie del subgénero Drosophila sin agrupar.

\section{MATERIALES Y MÉTODOS}

En diferentes años se han realizado recolecciones en Ecuador, el Páramo de Papallacta, provincia de Pichincha ( $0^{\circ} 20^{\prime} 09^{\prime}$ 'S, $78^{\circ} 12^{\prime} 32,1^{\prime \prime} \mathrm{W}$, altitud $\left.4.014 \mathrm{~m}\right)$, frente a la Laguna de Papallacta, provincia de Napo $\left(0^{\circ} 22^{\prime} 52,6^{\prime \prime} \mathrm{S}, 78^{\circ} 09^{\prime} 44,4^{\prime \prime} \mathrm{W}\right.$, altitud $\left.3.362 \mathrm{~m}\right)$ y en la Cordillera de las Guacamayos, provincia de Napo ( $0^{\circ} 37^{\prime} 08.7^{\prime}$ 'S, $77^{\circ} 50^{\prime} 21.2^{\prime \prime} \mathrm{W}$, altitud $\left.2.200 \mathrm{~m}\right)$. En todos los muestreos realizados se usaron trampas fabricadas con botellas de plástico agujereadas que se colgaron de los árboles a $1 \mathrm{~m}$ del suelo con cebo de banano y levadura. En el laboratorio las hembras fueron separas para fundar isolíneas. Los individuos recolectados fueron analizados tanto en su morfología externa como en su terminalia. Los términos morfológicos utilizados para las descripciones de las especies fueron tomados de BäCHLI et al. (2004). El índice del edeago fue tomado de ViLela (1983) y el índice de la espermateca de RAFAeL \& Arcos (1989). Los holotipos, alotipos y paratipos fueron disectados y montados en seco. La genitalia se colocó en un microtubo con glicerol. El ala derecha de 
los holotipos y alotipos se montó en una placa porta objetos con solución de tolueno. Todos los especímenes se depositaron en el Museo de Zoología, sección invertebrados, de la Pontificia Universidad Católica del Ecuador, Quito (QCAZ).

\section{RESULTADOS}

\section{Drosophila (Sophophora) neocapnoptera sp. nov.}

(Figs 1-10)

Material tipo. Holotipo (montado en seco, genitalia en microtubo), Ecuador, Napo, Cordillera de los Guacamayos (Carretera

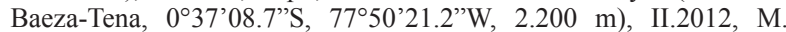
L. Figuero \& D. Céspedes col., M. L. Figuero \& V. Rafael det. (QCAZI 2725); alotipo + (montado en seco, genitalia en microtubo), los mismos datos del holotipo (QCAZI 2719); paratipos: 3 ㅇ, $5 \hat{\jmath}$ (montados en seco, genitalias en microtubos), los mismos datos del holotipo (QCAZI 2716- 2718, 2720- 2724)

Diagnosis. Cuerpo amarillento. Antenas con 6-7 ramas dorsales y 3-4 ventrales. Tórax con 7-8 hileras de pelos acrosticales entre las dorsocentrales anteriores. Alas con la vena transversal posterior ligeramente sombreada. Abdomen con diseño triangular. Edeago con espinas quitinizadas en la parte media central y microtricoso en los laterales. Ramas internas de las paráfisis bífidas.

Descripción del macho, holotipo y paratipos (individuos en alcohol y de isolínea). Morfología externa. Cuerpo amarillento. Longitud del cuerpo del holotipo: 3,5 $\mathrm{mm}$. Longitud del cuerpo del holotipo incluido alas: $3,8 \mathrm{~mm}$.

Cabeza. Color amarillo. Antenas amarillas, arista plumosa con 6 ramas dorsales y 3 ventrales más la terminal bifurcada (paratipos de 6 a 7 ramas dorsales y de 3 a 4 ventrales). Triángulo frontal amarillo, triángulo ocelar marrón, ocelos amarillos. Frontal vitta amarilla. Placa orbital amarilla, cerda orbital media más cerca a la anterior y al borde ocular. Ojos color rojo vinoso. Gena amarilla, carina amarilla no prominente, afilada (no surcada) y estrecha. Palpos amarillos.

Tórax. Color amarillo con 8 hileras de pelos acrosticales entre las dorsocentrales anteriores (paratipos de 7 a 8 hileras de pelos). Cerdas escutelares anteriores divergentes. Cerda catepisternal media más delgada que las otras dos. Longitud de la cerda media muy variable entre individuos (ie. en algunos individuos la cerda catepisternal media es casi imperceptible, es menos de $1 / 2$ de la anterior; en otros es más quitinizada y un poco más de $1 / 2$ de la anterior). Patas amarillas.

Alas. Amarillas, vena transversal posterior ligeramente sombreada. Longitud del ala del paratipo $2,51 \mathrm{~mm}$, ancho $1,16 \mathrm{~mm}$. Índices alares paratipo: alar $=2,15 ; \mathrm{C}=3,00 ; \mathrm{ac}=2,50 ; \mathrm{hb}=0,65 ; 4 \mathrm{c}=0,78 ; 4 \mathrm{v}=1,68$; $5 \mathrm{x}=1,33 ; \mathrm{M}=0,42$ y Prox $\mathrm{x}=0,42$. (Ala holotipo en mal estado).

Abdomen. Color amarillo. Primer segmento amarillo, del segundo al sexto con pigmentación en forma triangular en la parte media posterior. Pigmentación se adelgaza hacia los lados. Tercer y cuarto tergito, lateralmente la banda se difumina y alcanza el margen anterior.

Genitalia externa. Cercilibres, micropubescentes. Epandrio con 3 cerdas dorsales, 25 cerdas en el lóbulo ventral derecho y 23 en el izquierdo (paratipos de 25 a 27 cerdas). Surestilos alargados, el izquierdo presenta 14 y el derecho 15 dientes primarios dispuestos en forma de $\mathrm{S}$ (paratipos de 14 a 16 dientes primarios). En la parte inferior ambos surestilos presentan un diente más grande, una cerda larga hacia la parte interna y una corta hacia la parte externa (Fig. 1).

Genitalia interna. Hipandrio en forma de $\mathrm{V}$ poco quitinizado. Dorso-distalmente presenta dos apéndices muy quitinizados doblados y sub-apicalmente aserrados en el margen exterior. Gonopodios fusionados entre sí con una cerda pequeña en cada uno (Fig. 2). Apodema poco quitinizado, paráfisis (pinzas) fusionadas al apodema. Las 2 paráfisis divididas en la parte media en 2 ramas. Rama externa $3 / 4$ de la interna y muy quitinizada. Rama interna bífida y muy quitinizada en la punta, además presenta una proyección delgada y curva (Figs 3-5). Edeago tubular redondeado en la punta, en la parte anterior presenta dos prolongaciones laterales delgadas y curvadas ventralmente. Además presenta dos expansiones laterales poco quitinizadas, ligeramente curvadas en la parte media (alas). Parte media central del edeago con numerosas espinas quitinizadas y microtricoso en los lados (Figs 6-8). Índice del edeago= 1,41

Descripción de la hembra, alotipo y paratipos (individuos en alcohol y de isolínea). Morfología externa igual que el macho. Tórax de una de las hembras presenta la cerda catepisternal media muy quitinizada y más grande que la anterior.

Medidas e índices: longitud del cuerpo del alotipo: $3,7 \mathrm{~mm}$; longitud del cuerpo del alotipo incluido alas; 4 $\mathrm{mm}$; longitud del ala $2,65 \mathrm{~mm}$, ancho $1,12 \mathrm{~mm}$. Índices alares paratipo: alar $=2,35 ; \mathrm{C}=2,62 ; \mathrm{ac}=2,28 ; \mathrm{hb}=0,62$; $4 \mathrm{c}=0,80 ; 4 \mathrm{v}=1,70 ; 5 \mathrm{x}=1,28 ; \mathrm{M}=0,45$ y Prox $\mathrm{x}=0,50$.

Genitalia. Oviscapto poco quitinizado, alargado y terminado en punta. Presenta 19 ovisensillas como dientes marginales. Una ovisensilla subterminal larga y 3 ovisensillas como tricoides cortos distales (Fig. 9) (paratipos de 13 a 18 ovisensillas como dientes marginales). Espermatecas quitinizadas, elongadas con una invaginación que ocupa casi todo el largo de la espermateca. En la base presenta algunos pliegues (Fig. 10). Índice de la espermateca (base/altura) $=0,5$.

Relación con otras especies. Especie del grupo Drosophila willistoni emparentada con $D$. capnoptera (Tab. I).

Etimología. El prefijo neo, palabra proveniente del griego que significa novedad. La especie se denomina $D$. neocapnoptera sp. nov. por su descubrimiento posterior a D. capnoptera. 

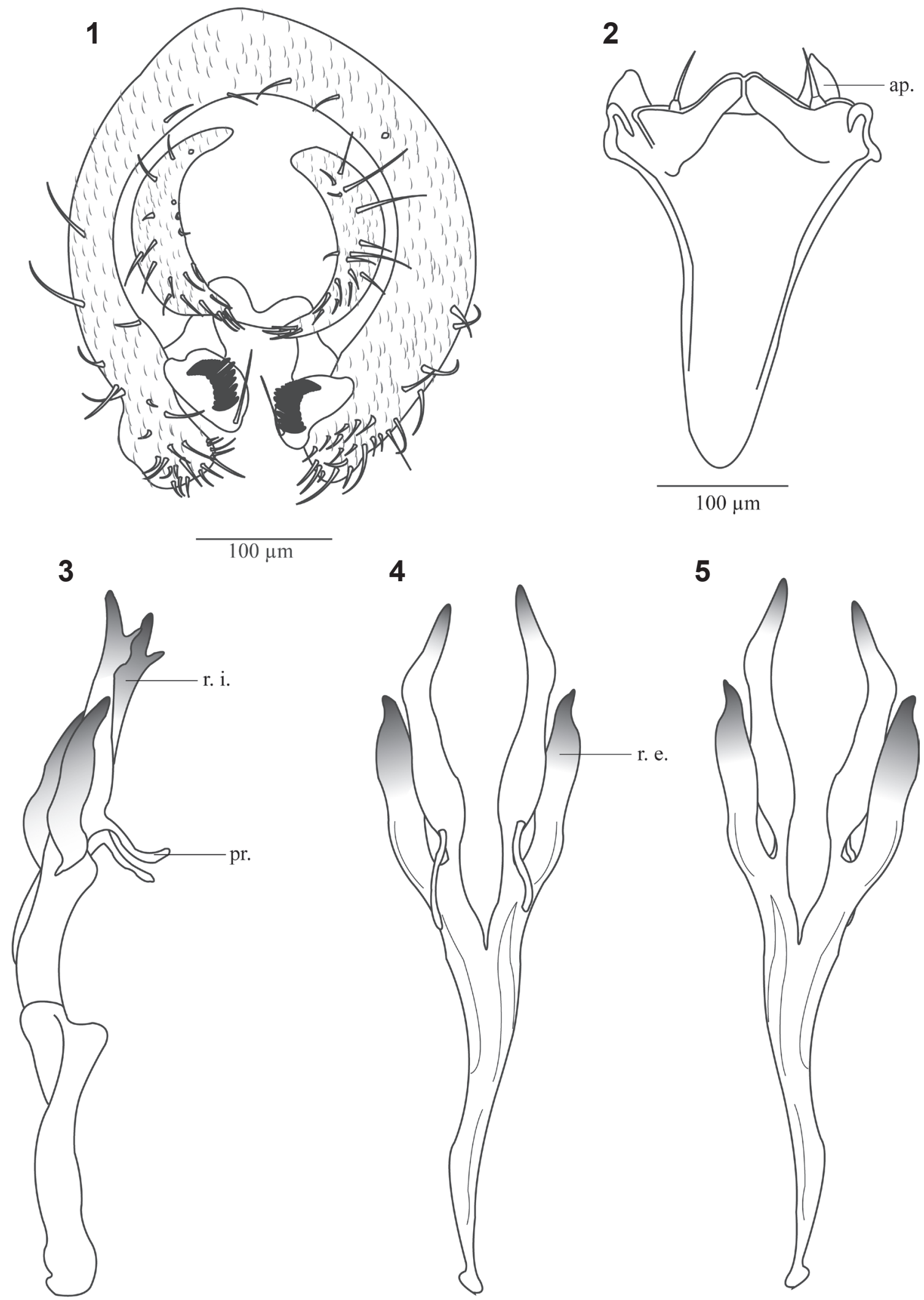

4

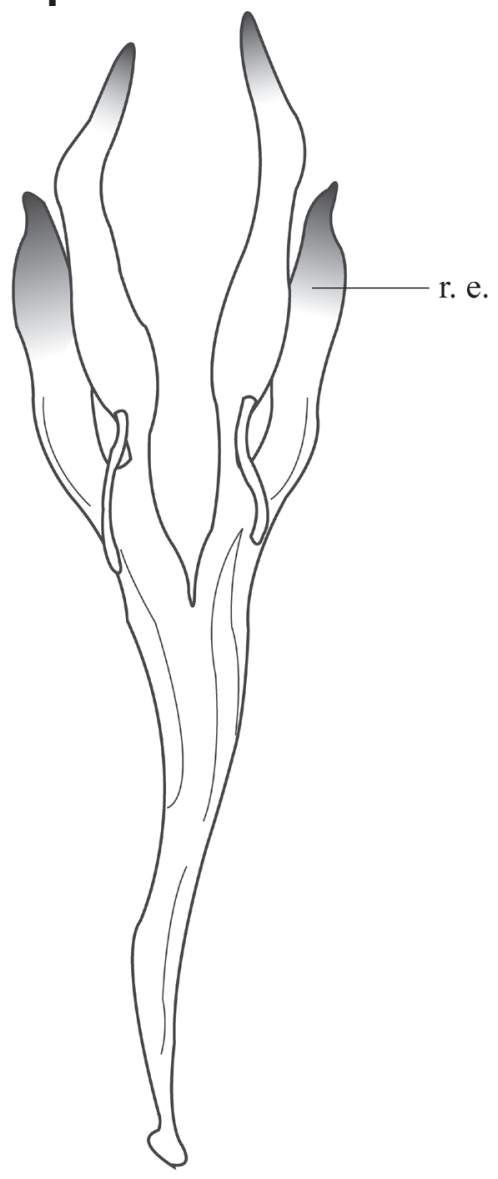

5

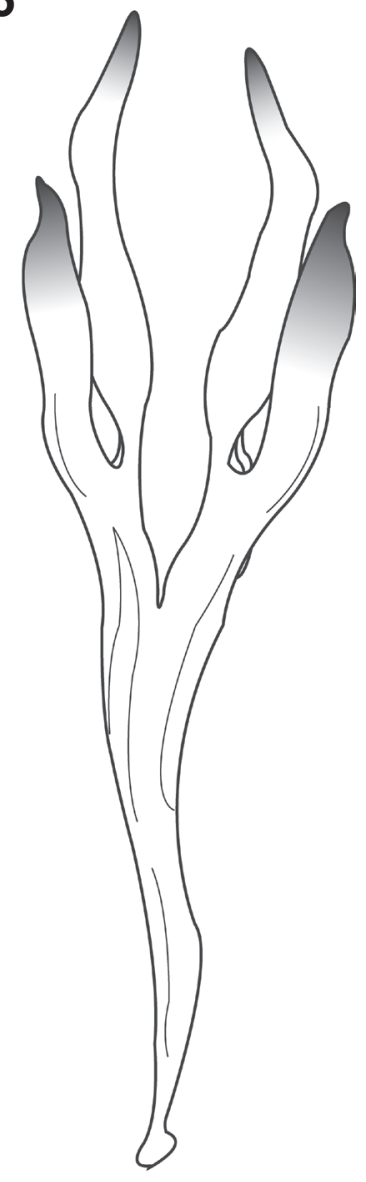

$100 \mu \mathrm{m}$

Figs 1-5. Drosophila (Sophophora) neocapnoptera sp. nov., holotipo ổ: 1, cerci; 2, hipandrio; 3-5, paráfisis y apodema en vista lateral, ventral y dorsal (ap., apéndices; pr., proyección; r.e., rama externa; r.i., rama interna). 

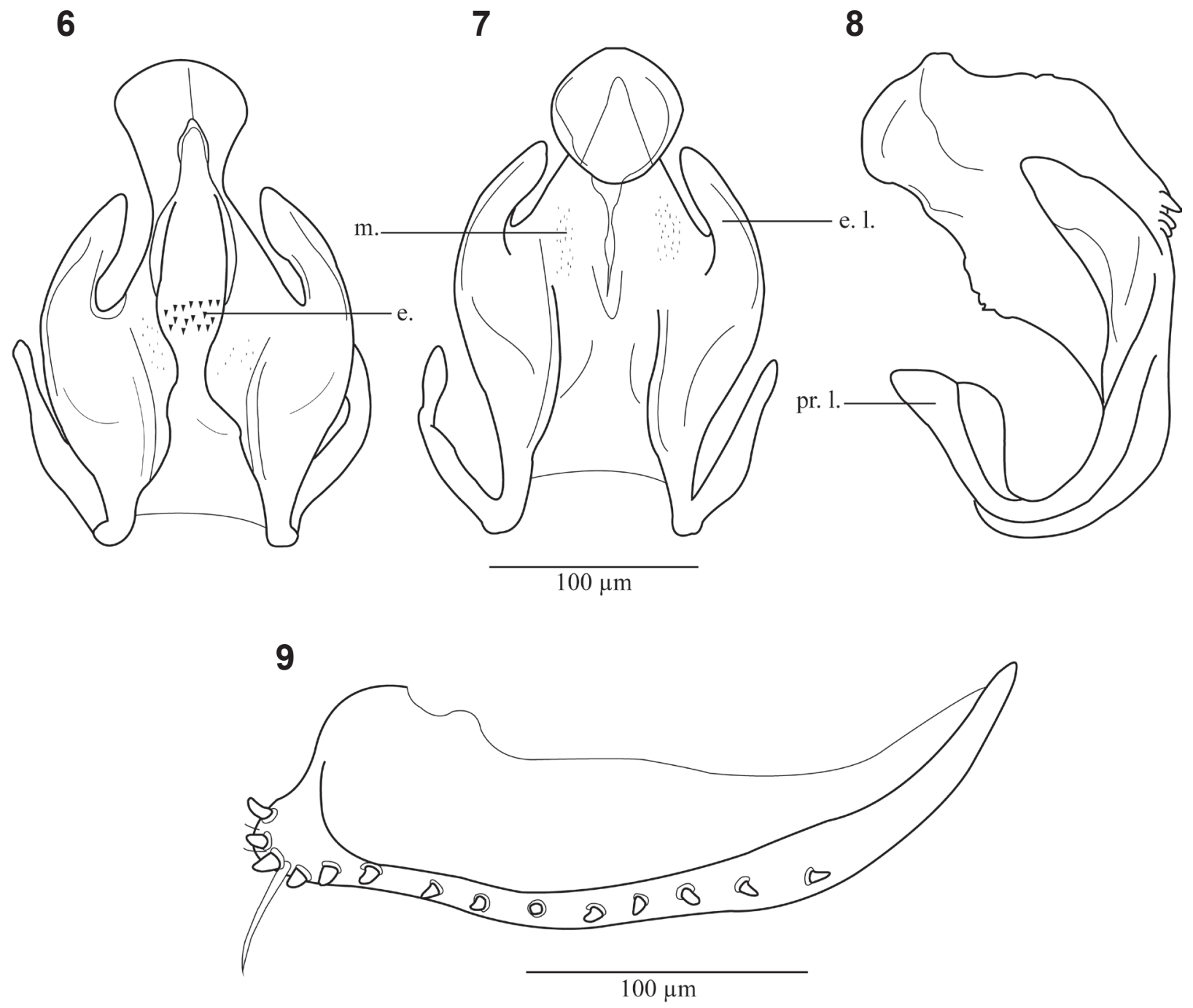

10

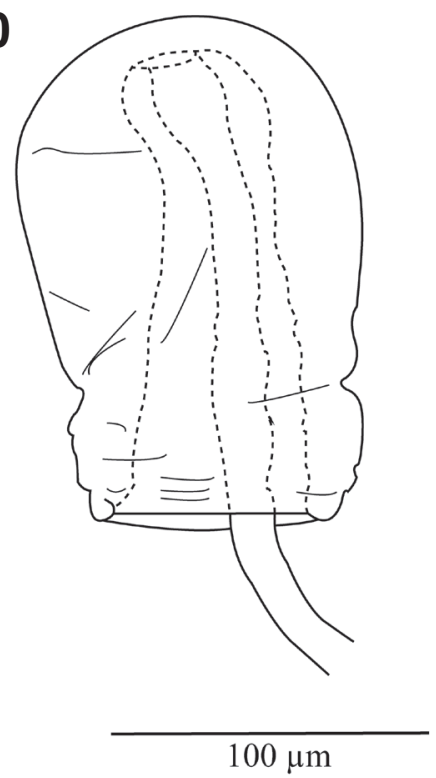

Figs 6-10. Drosophila (Sophophora) neocapnoptera sp. nov. 
Tab. I. Diferencias entre Drosophila capnoptera Patterson \& Mainland, 1944 (datos tomados de Vilela \& Báchli, 2002) y Drosophila neocapnoptera sp. nov., especies del grupo $D$. willistoni.

\begin{tabular}{|c|c|c|}
\hline & D. capnoptera ô & D. (S.) neocapnoptera sp. nov. $\hat{0}$ \\
\hline Antenas & $4-5$ ramas dorsales y 3 ventrales & 6-7 ramas dorsales y 3-4 ventrales \\
\hline Ala & $\begin{array}{c}\text { área marrón claro en la mitad de la vena costal cruzando } \\
\text { la vena R4+5 en la mitad apical; área triangular negra que } \\
\text { se extiende desde R1 hasta vena transversal R-M; vena } \\
\text { transversal DM-Cu sombreada }\end{array}$ & $\begin{array}{c}\text { sin pigmentación, vena transversal posterior ligeramente } \\
\text { sombreada }\end{array}$ \\
\hline Hipandrio & ancho & delgado \\
\hline Edeago & prominencia dorsal y dentada en el ápice & $\begin{array}{c}\text { sin prominencia, espinas en la parte dorsal visibles en } \\
\text { vista lateral }\end{array}$ \\
\hline Paráfisis externa & delgados & anchos y muy quitinizados en la punta \\
\hline Paráfisis interna & poco quitinizadas & muy quitinizadas en la punta \\
\hline Distribución & $\begin{array}{l}\text { México, El Salvador, Honduras, Nicaragua, Costa Rica, } \\
\text { Panamá }\end{array}$ & Ecuador \\
\hline
\end{tabular}

\section{Drosophila (Drosophila) neoasiri sp. nov.} (Figs 11-15)

Material tipo. Holotipo o (disectado, montado en seco, genitalia en microtubo), Ecuador, Napo, Papallacta (Laguna de Papallacta, $0^{\circ} 22^{\prime} 52,6$ '’S, 7809’44,4’'W, 3.362 m), I.2006, V. Rafael

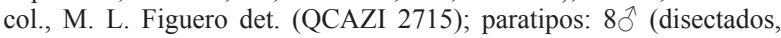
montados en seco, genitalias en microtubo), los mismos datos del holotipo (QCAZI 2707- 2714).

Diagnosis. Longitud del cuerpo incluido alas: 6,5$7,0 \mathrm{~mm}$. Tórax color marrón con una franja más oscura en la mitad. Abdomen con pigmentación rectangular más oscura en la parte central. Surestilos redondeados y micropubescentes con 8-11 dientes primarios, 1517 cerdas marginales y 1-2 cerdas como dientes. Edeago quitinizado y ancho, en la parte dorsal con dos proyecciones laterales quitinizadas como cuernos y fuertemente aserrado en la parte ventral. En vista lateral de forma globosa.

Descripción del macho, holotipo y paratipos (individuos en alcohol). Morfología externa. Longitud del cuerpo del holotipo incluido alas: $6,5 \mathrm{~mm}$. Longitud del cuerpo de los paratipos incluido alas: 6,5-7,0 $\mathrm{mm}$. Color del cuerpo marrón.

Cabeza. Primer segmento de las antenas marrón oscuro, segundo segmento marrón claro. Arista plumosa con 4 ramas dorsales y 2 ventrales (algunos paratipos con 5 ramas dorsales) y bifurcación terminal. Placa orbital marrón; cerda orbital media más cerca de la cerda orbital anterior y al borde ocular. Triángulo ocelar marrón oscuro, ocelos amarillos. Triángulo frontal y frontal vitta marrón. Carina poco prominente marrón y ligeramente surcada. Proboscis y palpos amarillentos. Ojos rojizos.

Tórax. Marrón con una franja más oscura en el centro que ocupa el espacio entre dos filas de pelos acrosticales (en paratipos la franja es más ancha). Seis hileras de pelos acrosticales entre las cerdas dorsocentrales anteriores (paratipos de 6 a 7 hileras de pelos). Escutelo del mismo color que el tórax, cerdas escutelares anteriores divergentes. Katepisterno amarillo, cerda esternopleural media pequeña, 1/4 de la anterior o más pequeña. Patas amarillas, últimos segmentos tarsales marrón oscuro.

Alas. Amarillas con la vena transversal posterior sombreada. Celda marginal, submarginal, primera celda posterior y primera basal ligeramente más oscuras. Longitud del ala $5,02 \mathrm{~mm}$. Índices alares: alar $=2,42$; $\mathrm{C}=4,76 ; \mathrm{ac}=1,75 ; \mathrm{hb}=0,24 ; 4 \mathrm{c}=0,49 ; 4 \mathrm{v}=1,32 ; 5 \mathrm{x}=1,07$; $\mathrm{M}=0,32$ y $\operatorname{Prox} \mathrm{x}=0,44$.

Abdomen. Marrón amarillento, sin línea media dorsal, tergitos con pigmentación más oscura en la parte central de forma rectangular o de reloj de arena.

Genitalia externa. Cerci micropubescentes con cerdas, y parcialmente unido al epandrio. Epandrio micropubescente. Surestilos redondeados y micropubescentes. Surestilo derecho con 8 dientes primarios separados es $5-2-1,17$ cerdas marginales y 2 cerdas como dientes; surestilo izquierdo con 9 dientes primarios separados en 6-1-2, 18 cerdas marginales y 1 cerda como diente (paratipos de 7 a 11 dientes primarios, 15 a 18 cerdas marginales y de 1 a 2 cerdas como dientes) (Fig. 11).

Genitalia interna. Hipandrio del mismo tamaño que el epandrio, en forma de U. Arco ausente. Gonopodios de forma rectangular con una cerda muy larga (Fig. 12). Edeago quitinizado y ancho. Parte dorsal con dos proyecciones laterales muy quitinizadas como cuernos que nacen en la parte apical y dirigidos hacia la parte anterior. En vista lateral de forma globosa como $D$. asiri y ventralmente con una estructura membranosa, fuertemente aserrado. Rama ventral del edeago larga y quitinizada. Paráfisis redondas con una cerda larga, una espina y microtricosa en la parte ventral. Apodema del edeago poco quitinizado (Figs 13-15). Índice del edeago $=1,03$.

Relación con otras especies. Especie del grupo D. asiri, emparentada con D. asiri.

Etimología. El prefijo neo, palabra proveniente del griego, que significa novedad. La especie se denomina $D$. neoasiri sp. nov. por su descubrimiento posterior a $D$. asiri. 
11

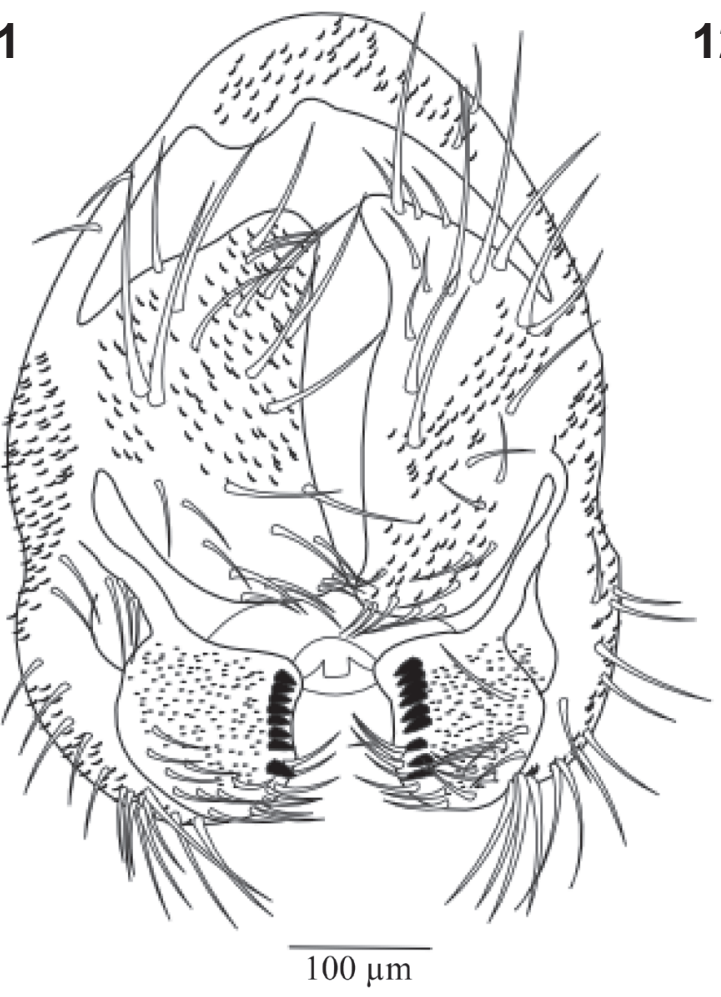

12

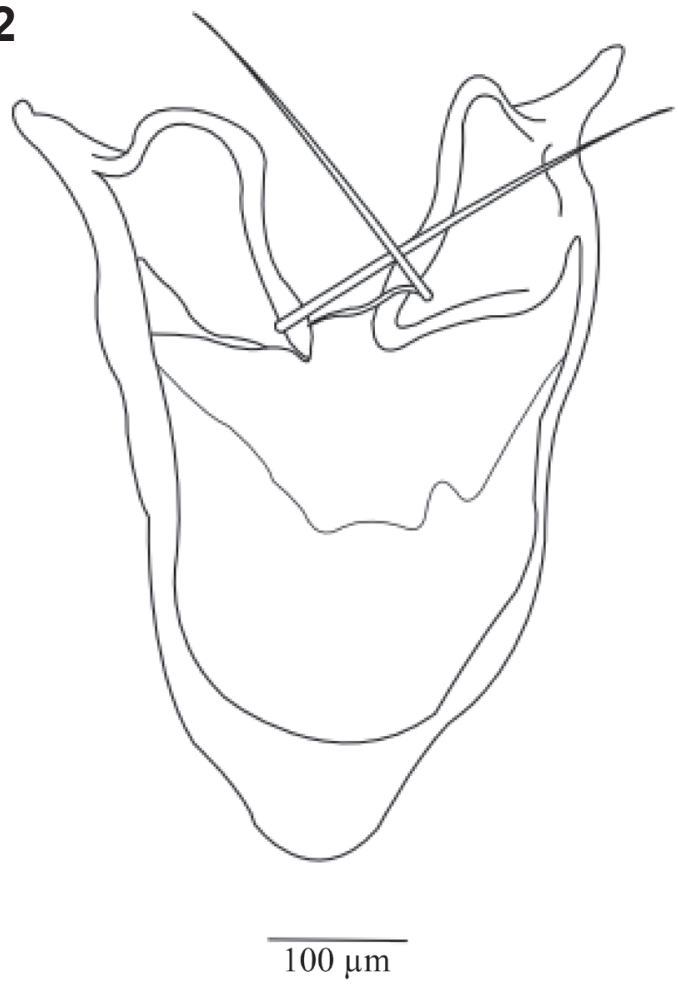

13

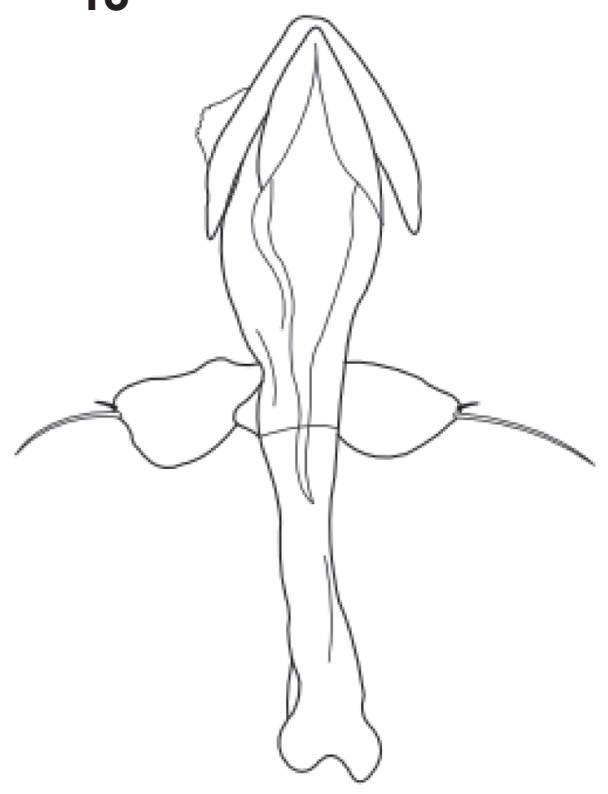

14

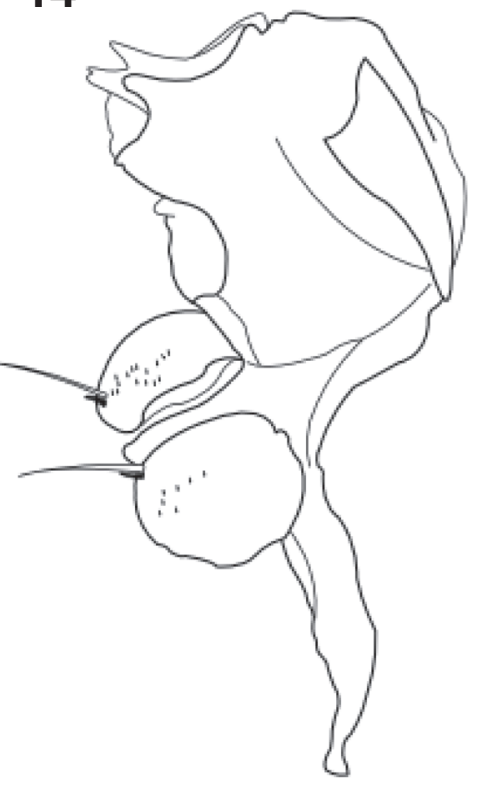

15

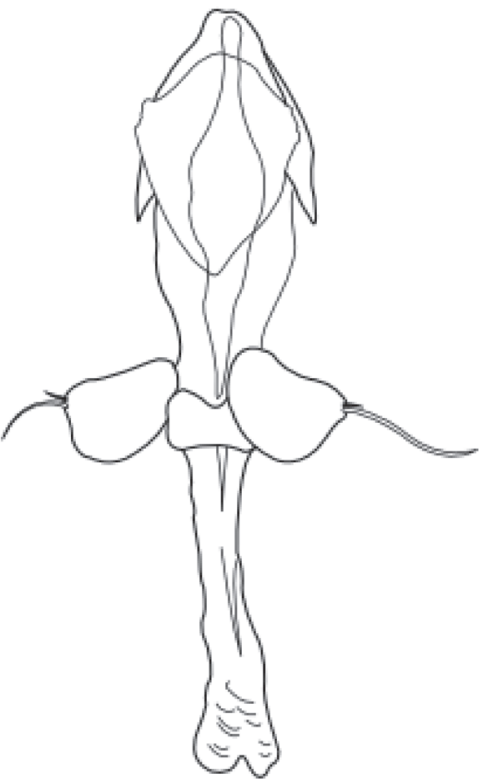

$100 \mu \mathrm{m}$

Figs 11-15. Drosophila (Drosophila) neoasiri sp. nov., holotipo ổ: 11, cerci; 12, hipandrio; 13-15, edeago en vista dorsal, lateral y ventral.

Drosophila (Drosophila) papallacta sp. nov.

(Figs 16-24)

Material tipo. Holotipo $\widehat{\partial}$ (disectado, montado en seco, genitalia en microtubo), Ecuador, Pichincha, Papallacta (Páramo de Papallacta, $0^{\circ} 20^{\prime} 09,4^{\prime}$ 'S, 78 $\left.12^{\prime} 32,1^{\prime \prime} \mathrm{W}, 4.014 \mathrm{~m}\right)$, IV.2009, M. L. Figuero col., M. L. Figuero det. (QCAZI 2235); alotipo (disectado, montado en seco, genitalia en microtubo) los mismos datos del holotipo (QCAZI 2246); paratipos: 67,7 (disectados, montados en seco, genitalias en microtubo) los mismos datos del holotipo; II, IV, VII, IX.2009, M. L. Figuero col. (QCAZI 2236-2238, 2241-2243, 2248, 2250-2252, 2254-2256). Paratipos: $4 \hat{\gamma}, 3$ (disectados, montados en seco, genitalias en microtubo), Ecuador, Pichincha, Papallacta (Páramo de Papallacta, 0¹9’24,8’S, 78¹2’42,8’W, $4.005 \mathrm{~m})$, IV, VII, IX.2009, M. L. Figuero col., M. L. Figuero det. (QCAZI $2239,2240,2244,2245,2247,2249,2253)$. 
Diagnosis. Alas marrón claro con la vena transversal posterior sombreada. Abdomen con pigmentación de forma rectangular o como reloj de arena. Cerci parcialmente fusionado al epandrio. Surestilos con 10-13 dientes primarios y 30 cerdas marginales. Edeago con pequeñas proyecciones puntiagudas en los extremos laterales de la cabeza; parte dorsal con una estructura como joroba (proceso dorsal). Oviscapto con 22-28 ovisensillas como dientes. Espermateca quitinizada con una invaginación que alcanza $3 / 4$ de la espermateca. Además presenta una depresión membranosa como hueco en la parte media distal.

Descripción del macho, holotipo (individuo en seco) y paratipos (individuos en alcohol). Morfología externa. Longitud del cuerpo del holotipo incluido alas: $5,5 \mathrm{~mm}$; longitud del cuerpo de los paratipos incluido alas: 5,5-6,0 mm. Color del cuerpo marrón claro.

Cabeza. Primer y segundo segmento de las antenas marrón claro; arista plumosa con 3 ramas dorsales y 2 ramas ventrales, más la bifurcación terminal. Cabeza marrón claro (otros individuos presentan la cabeza más oscura). Placa orbital marrón claro; cerda orbital media más cerca de la cerda orbital anterior y al borde ocular. Triángulo ocelar marrón, ocelos amarillos. Triángulo frontal marrón, frontal vitta marrón claro. Cerda oral gruesa y larga. Carina poco prominente de color marrón claro y ligeramente surcada, en las partes más prominentes de la carina presenta una franja oscura. Proboscis y palpos amarillentos. Ojos de color marrón rojizo.

Tórax. Marrón claro sin diseño con 6 hileras de pelos acrosticales entre las dorsocentrales anteriores (paratipos de 6 a 7 hileras de pelos). Escutelo del mismo color que el tórax con cerdas escutelares anteriores divergentes. Cerda esternopleural media $1 / 3$ de la anterior. Patas amarillas, los dos últimos segmentos tarsales marrón oscuros.

Alas. Color marrón claro. Casi a todo lo largo de la vena radial, la parte distal de la primera celda basal, la mitad inferior de la celda discal y la segunda y tercera celda posterior son zonas más claras, la vena transversal posterior sombreada (Fig. 16). Longitud del ala 3,86 mm. Índices alares: $a l a r=2,15 ; \mathrm{C}=5 ; \mathrm{ac}=1,25 ; \mathrm{hb}=0,5$; $4 \mathrm{c}=0,5 ; 4 \mathrm{v}=1,38 ; 5 \mathrm{x}=0,78 ; \mathrm{M}=0,30$ y Prox $\mathrm{x}=0,66$.

Abdomen. Color marrón amarillento, tergitos con pigmentación oscura en la parte media, de forma rectangular o de reloj de arena (algunos paratipos con línea oscura a lo largo de la parte inferior de cada tergito).

Genitalia externa. Cerci micropubescentes con cerdas, y parcialmente unido al epandrio. Epandrio micropubescente, con 3 cerdas en la parte dorso lateral de cada lado; en el lóbulo ventral se agrupan más de 20 cerdas y presenta una estructura quitinizada y desnuda que se proyecta sobre los surestilos. Decasterno rectangular con la parte anterior curva y la posterior muy quitinizada (Fig. 17). Surestilos rectangulares y micropubescentes en la cara externa de los dientes primarios; presentan 12 dientes primarios y numerosas cerdas marginales muy largas, aproximadamente 30 en ambos lados (algunos paratipos con dientes primarios separados por un espacio y pueden tener de 10 a 13 dientes primarios) (Fig. 18).

Genitalia interna. Hipandrio del mismo tamaño que el epandrio, en forma de U, quitinizado en la parte anterior. Gonopodios de forma ovalada y estriados; parte anterior del gonopodio con una cerda con la punta curvada (Fig. 19). Edeago quitinizado, ventralmente de forma triangular con la punta ligeramente bífida y apenas curvada dorsalmente. En vista lateral, pequeñas proyecciones puntiagudas quitinizadas en los extremos laterales del edeago y en la parte dorsal presenta una estructura que se asemeja a una joroba o proceso dorsal, quitinizada. Rama ventral del edeago corta y muy quitinizada. Paráfisis circular con 1 cerda y 3 pequeñas espinas en la parte media (paratipos de 1 a 2 cerdas y de 1 a 3 espinas). Apodema del edeago quitinizado excepto en su parte distal (Figs 20-22). Índice del edeago=1,4.

Descripción de la hembra, alotipo y paratipos (individuos en alcohol). Morfología externa igual que el macho.

Medidas e índices: longitud del cuerpo del alotipo incluido ala $6,0 \mathrm{~mm}$; longitud del cuerpo de los paratipos incluido alas: 5,5-6,1 $\mathrm{mm}$; longitud del ala $4,3 \mathrm{~mm}$. Índices alares: $\mathrm{alar}=2,07 ; \mathrm{C}=4,47 ; \mathrm{ac}=1,18 ; \mathrm{hb}=0,34$; $4 c=0,57 ; 4 v=1,42 ; 5 x=0,89 ; M=0,37$ y Prox $x=0,69$.

Genitalia. Oviscapto alargado y quitinizado, amarillo. Oviscapto con 20 ovisensillas como dientes marginales y 6 ovisensillas como dientes discales, 1 ovisensilla terminal larga y 3 ovisensillas tricoidales distales (paratipos de 22-28 ovisensillas como dientes, 1 ovisensilla terminal larga y de 1 a 4 ovisensillas tricoidales distales) (Fig. 23). Espermateca quitinizada, redondeada, con invaginación que alcanza $3 / 4$ de la espermateca. La parte proximal más estrecha, con pliegues; en la parte distal, pequeñas porosidades. La parte media distal presenta una depresión membranosa dando la apariencia de un hueco; a esta depresión se le une una membrana interna que se extiende hacia la parte proximal uniéndose a la invaginación (Fig. 24). Índice de la espermateca $=0,93$.

Etimología. Papallacta, palabra del idioma quichua que significa tierra de la papa. El nombre de la especie nueva hace referencia al lugar de captura.

\section{DISCUSIÓN}

Drosophila neocapnoptera sp. nov. es similar a $D$. capnoptera sin embargo presentan algunas diferencias que permiten distinguirlas. Además de ser especies de diferentes países, se las puede identificar porque $D$. capnoptera presenta pigmentación en varias zonas del ala, mientras que $D$. neocapnoptera sp. nov. tiene una ligera sombra en la vena transversal posterior. Por otro lado $D$. neocapnoptera sp. nov. se pudo reproducir en el laboratorio, como muchas otras especies del grupo D. willistoni (ie. D. capricorni Dobzhansky \& Pavan, 1943; D. willistoni Sturtevant, 1916) por lo que se pudo identificar fácilmente a la hembra. Al igual que 
16

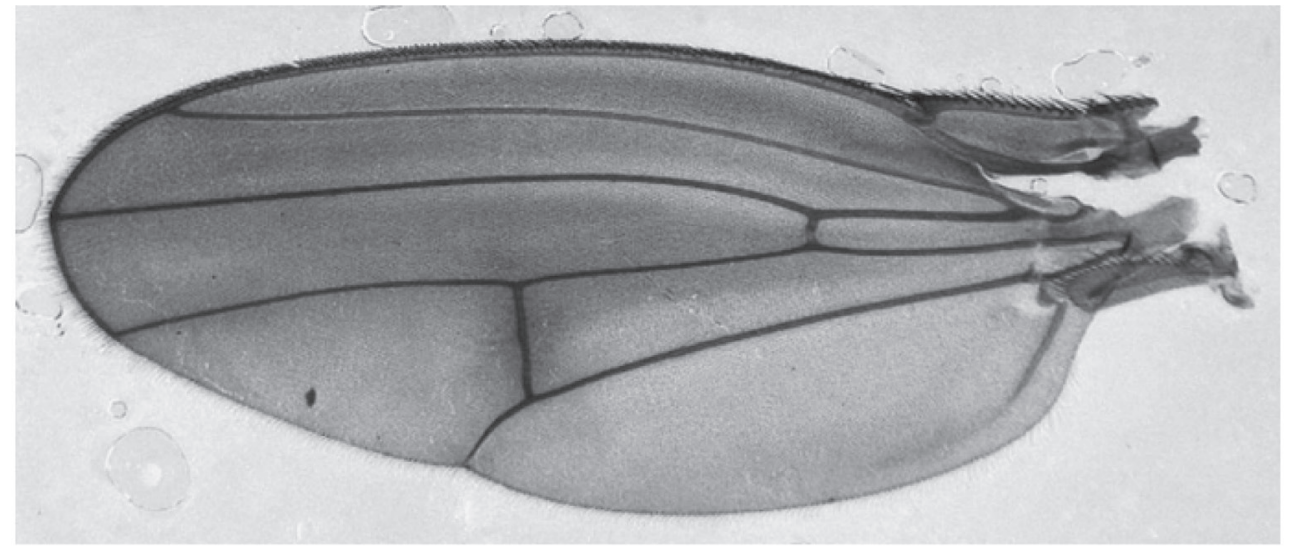

18

17
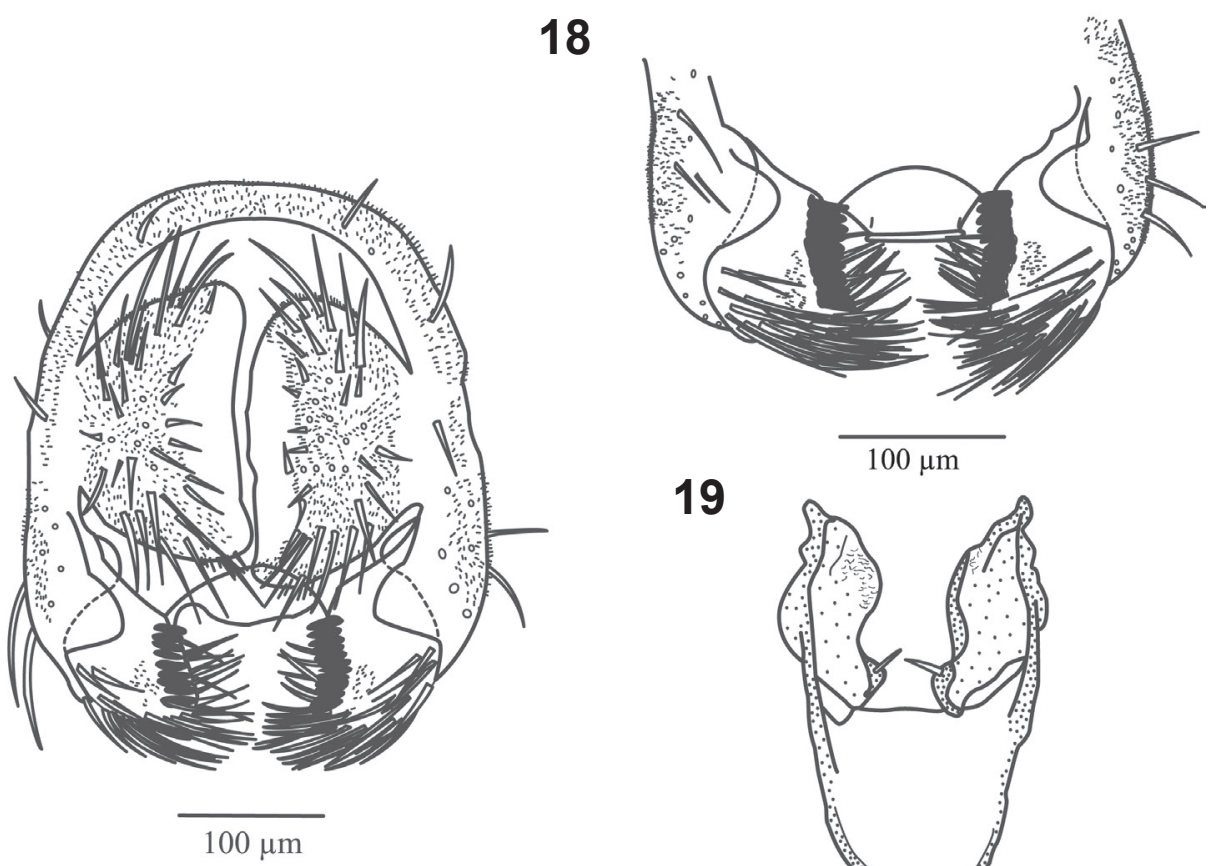

19

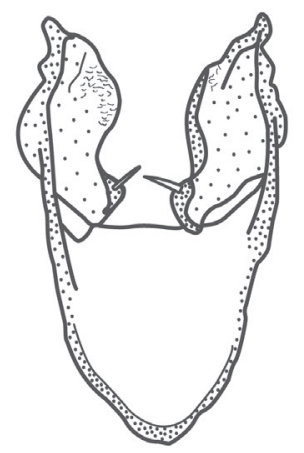

$\overline{100 \mu \mathrm{m}}$
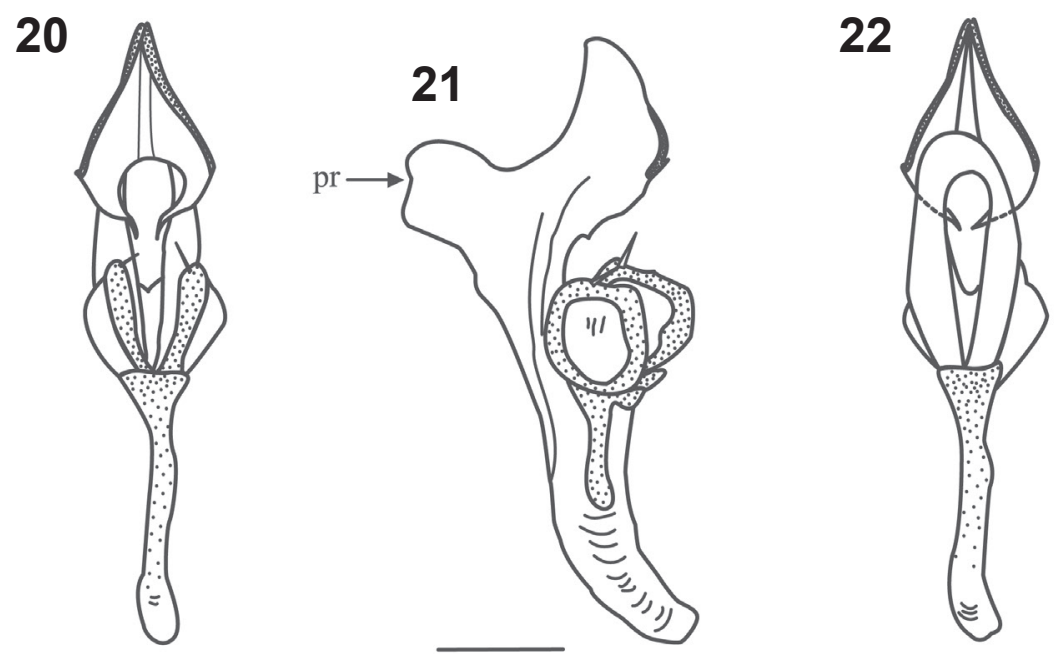

$100 \mu \mathrm{m}$

Figs. 16-22. Drosophila (Drosophila) papallacta sp. nov., holotipo 3 : 16, ala derecha; 17, cerci; 18, surestilos; 19, hipandrio; 20-22, edeago en vista ventral, lateral y dorsal (pr, proceso dorsal). 

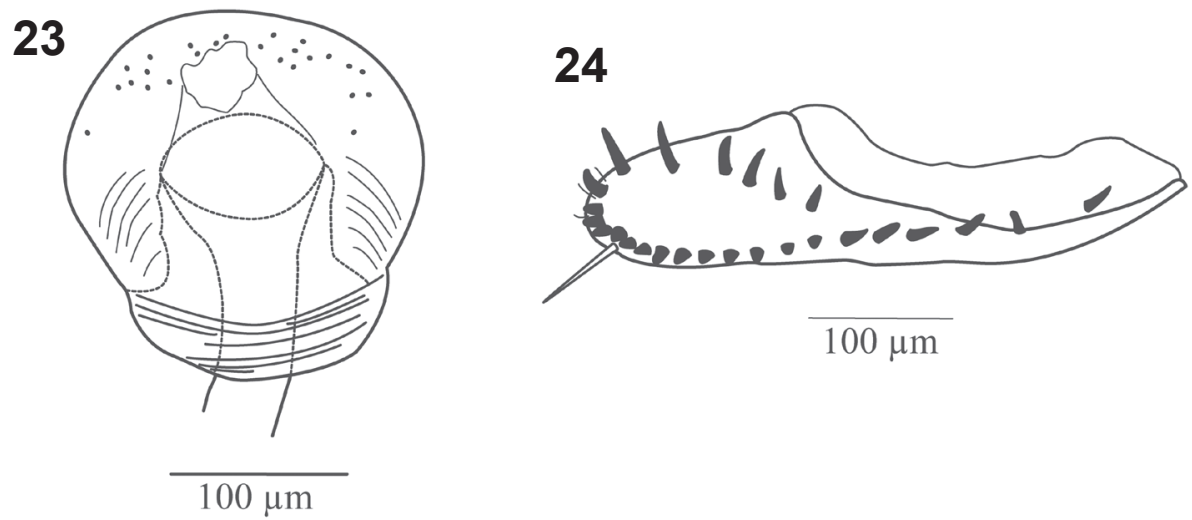

Figs. 23, 24. Drosophila (Drosophila) papallacta sp. nov., alotipo $ๆ: 23$, oviscapto; 24, espermateca.

D. capnoptera, D. neocapnoptera sp. nov. pertenecería al complejo de especies alagitans-bocainensis, que se distinguen de las otras especies del grupo $D$. willistoni por las características de las pinzas, ya que presentan bifurcación apical, ramas laterales y ramas secundarias (WheELER \& MAGALhãES, 1962).

La genitalia de los machos, especialmente el edeago, es el carácter más significativo y usado por los taxónomos para identificar a las especies (VILELA \& BäCHLI, 1990). Este es el caso de $D$. neoasiri sp. nov., similar a $D$. asiri, la diferencia más relevante entre las dos especies se observa a nivel del edeago. En D. neoasiri sp. nov. el aserrado en vista lateral es más conspicuo que en $D$. asiri; esta diferencia en la forma del edeago es suficiente como para discriminarlas.

Drosophila papallacta sp. nov. por el momento no se encuentra relacionada a ningún grupo de especies de Drosophila, aunque presenta características morfológicas comunes con las especies del grupo D. mesophragmatica; es necesario realizar estudios filogenéticos a nivel morfológico y molecular para conocer la relación de esta especie a un grupo específico.

La distribución de $D$. neoasiri sp. nov. y de $D$. papallacta sp. nov. en el Ecuador, está restringida a los bosques andinos y páramos. ATKINSON (1994) propone que los organismos ectotermos expuestos a bajas temperaturas presentan un aumento en el tamaño del cuerpo. $D$. neoasiri sp. nov. y $D$. papallacta sp. nov. son especies de tamaño grande (6,5 y 5,5 $\mathrm{mm}$ respectivamente), en comparación a especies como D. melanogaster Meigen, 1830 (2-3 mm) (SHORROCKS, 1972), lo que plantearía que estas especies están adaptadas a las condiciones climáticas de altura.

Agradecimientos. A la Pontifica Universidad Católica del Ecuador por financiar los proyectos No. E29181 y No. I13058. Al Ministerio de Ambiente por emitir la autorización de investigación científica No. 008-09 IC-FAU-DNB/MA. Al personal del laboratorio de Genética Evolutiva. Al licenciado Diego Céspedes por su ayuda en las recolectas y con los dibujos y al licenciado Christian Samaniego por la ayuda con la traducción del resumen. A Idea Wild por la donación de materiales.

\section{REFERENCIAS BIBLIOGRÁFICAS}

Acurio, A. E. \& RAfael, V. L. 2009. Inventario taxonómico de
Drosophilidae (Diptera) en el parque Nacional Yasuní, Amazonía Ecuatoriana. Acta Amazonica 39(3):713-718.

ATKINSON, D. 1994. Temperature and organism size-a biological law of ectotherms? Advances in Ecological Research 25:1-58.

Bächli, G.; Vilela, C. R.; Escher, S. A. \& Saura, A. 2004. The Drosophilidae (Diptera) of Fennoscadia and Denmark. Fauna Entomologica Scandinavica 39. Leiden, Boston, Brill. 362p.

Brake, I. \& Bächli, G. 2008. Drosophilidae (Diptera). In: World Catalogue of Insects 9:1-412. Stenstrup, Apollo Books.

CÉSPedes, D. \& Rafael, V. 2012. Descripción de una nueva especie del género Drosophila (Diptera, Drosophilidae) miembro del grupo de Drosophila tripuctata. Revista Ecuatoriana de Medicina y Ciencias Biológicas 33(1-2):124-128.

Figuero, M. L. \& Rafael, V. 2011. Dos nuevas especies del grupo Drosophila onychophora (Diptera, Drosophilidae) en los bosques de Polylepis de Papallacta, Pichincha, Ecuador. Iheringia, Série Zoologia 101(4):342-349.

Figuero, M. L.; RAFAel, V. \& CÉSPedes, D. 2012a. Grupo Drosophila asiri (Diptera, Drosophilidae), un nuevo grupo de especies andinas con la descripción de dos nuevas especies y la redescripción de Drosophila asiri. Iheringia, Série Zoologia 102(1):33-42.

Figuero, M. L.; León, R.; RAfael, V. \& CéSPedes, D. $2012 \mathrm{~b}$. Cuatro nuevas especies del grupo Drosophila onychophora (Diptera, Drosophilidae) en el Parque Arqueológico Rumipamba, Pichincha, Ecuador. Iheringia, Série Zoologia 102(2):212-220.

O'Grady, P. M. \& Kidwell, M. G. 2002. Phylogeny of the subgenus Sophophora (Diptera, Drosophilidae) based on combined analysis of nuclear and mitochondrial sequences. Molecular Phylogenetics and Evolution 22(3):442-453.

Rafael, V. \& ArCos, G. 1989. Subgrupo inca, un nuevo subgrupo del grupo repleta, con descripción de Drosophila huancavilcae nov. sp. (Diptera, Drosophilidae). Evolución Biológica 3(3):233-243.

Shorrocks, B. 1972. Invertebrate Types. Drosophila. London, Ginn \& Company Limites. 144p.

Sturtevant, A. H. 1939. On the subdivision of the genus Drosophila. Proceedings of the National Academy of Sciences of the United States of America 25:137-141.

1942. The classification of the genus Drosophila with the description of nine new species. The University of Texas Publication 4213:5-51.

VILELA, R. C. A. 1983. Revision of the Drosophila replete species group (Diptera, Drosophilidae). Revista Brasileira de Entomologia 27(1):78-81.

Vilela, C. \& BëCHLI, G. 1990. Taxonomic studies on Neotropical species of seven genera of Drosophilidae (Diptera). Bulletin de la Société Entomologique Suisse 63(Suppl.):1-332.

2002. On the identity of four poorly known species of Neotropical Drosophilidae (Diptera). Mitteilungen der Schweizerischen entomologischen Gesellschaft, Neuchâtel 75(3-4):197-210.

WheELER, M. R. \& MAGALhãES, L. E. 1962. The alagitans-bocainensis complex of the willistoni group of Drosophila. Studies in Genetics II. The University of Texas Publications 6205:155-173. 